\title{
The evolving landscape of predictive biomarkers of response to PARP inhibitors
}

\author{
Anish Thomas, Junko Murai, and Yves Pommier \\ Developmental Therapeutics Branch and Laboratory of Molecular Pharmacology, Center for Cancer Research, National Cancer Institute, Bethesda, Maryland, USA.
}

\begin{abstract}
Poly(ADP-ribose) polymerase inhibitors (PARPis) are DNA-damaging agents that trap PARP-DNA complexes and interfere with DNA replication. Three PARPis - olaparib, niraparib, and rucaparib - were recently approved by the FDA for the treatment of breast and ovarian cancers. These PARPis, along with 2 others (talazoparib and veliparib), are being evaluated for their potential to treat additional malignancies, including prostate cancers. While lack of PARP-1 confers high resistance to PARPis, it has not been established whether or not the levels of PARP-1 directly correlate with tumor response. In this issue of the $J C l$, Makvandi and coworkers describe an approach to address this question using $\left[{ }^{18} \mathrm{~F}\right]$ FluorThanatrace, an $\left[{ }^{18} \mathrm{~F}\right]-$ labeled PARP-1 inhibitor, for PET. The tracer was taken up by patient tumor tissue and appeared to differentiate levels of PARP-1 expression; however, future studies should be aimed at determining if this tracer can be used to stratify patient response to PARPi therapy.
\end{abstract}

Three PARPis are now licensed for use as single agents: olaparib for patients with germline BRCA-mutated breast and advanced ovarian cancers who have previously been treated with chemotherapy (6); rucaparib for patients with germline and/ or somatic BRCA-mutated advanced ovarian cancer treated previously with chemotherapy (7); and niraparib, which along with olaparib is approved as maintenance therapy regardless of $B R C A$ mutation in patients with recurrent epithelial ovarian, fallopian tube, or primary peritoneal cancer who are in a complete or partial response to platinum-based chemotherapy $(8,9)$. Two additional PARPis, talazoparib and veliparib, are currently in advanced phase clinical trials.

PARPis differ in their PARP-trapping potency (Figure 1). While talazoparib is the most potent PARP trapper (nanomolar potency), veliparib is primarily a catalytic inhibitor with only weak PARP-trapping ability $(5,10)$. Hence, the antitumor activity of veliparib is limited as a single agent, and this PARPi is mostly active in combination with TOP1 inhibitors (11) and radiotherapy (12). The other PARPis primarily act as DNA-damaging agents by PARP trapping, with talazoparib having the greatest potency, followed by niraparib, olaparib, and rucaparib, which have similar potency (Figure 1A) $(5,10)$.

Although many patients are benefiting from PARPi therapy, predicting an individual's response to treatment remains imprecise. Mutations in BRCA genes, which are essential for highfidelity repair of DNA double-strand breaks through the homologous recombination (HR) repair pathway, do not entirely account for the treatment benefit associated with PARPis. Measures of HR deficiency (HRD) also are not sufficiently precise to predict which patients will respond, and recent trials have shown that PARPi therapy can be beneficial to patients without known HRD (13). It is clear from studies in cancer cell lines and 
A

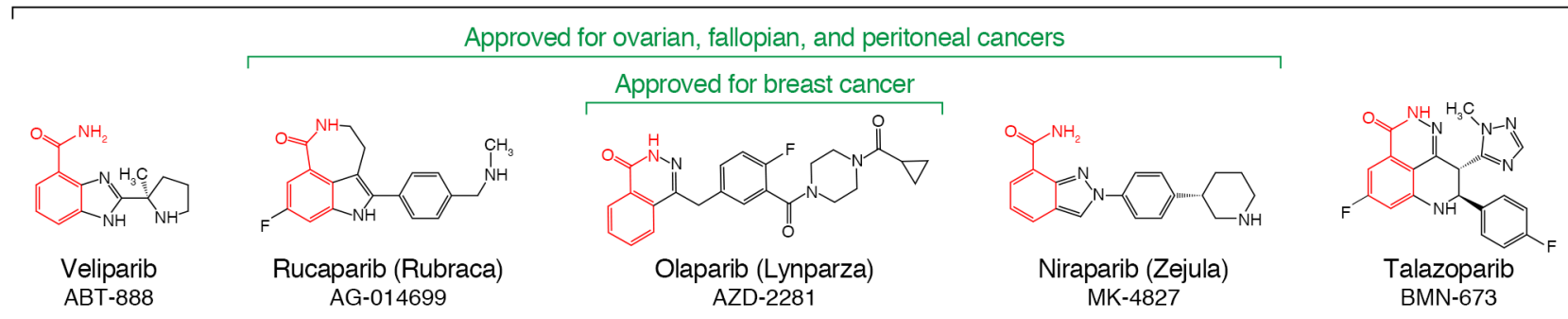

PARP trapping potency

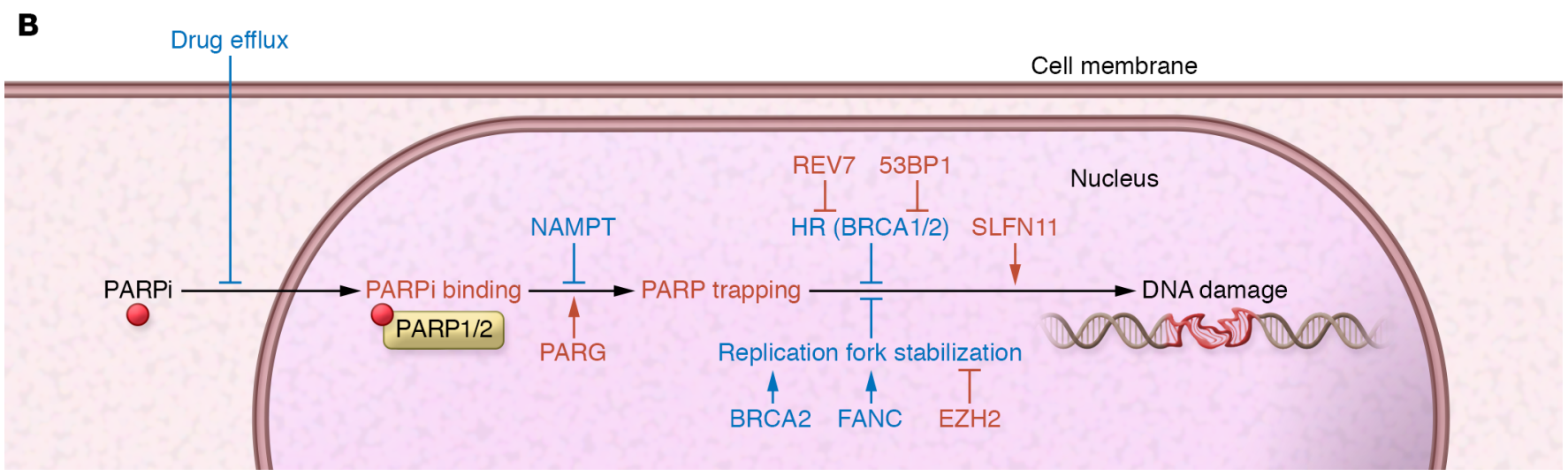

Figure 1. Clinical PARP inhibitors (PARPis) and determinants of response and resistance to PARPis. (A) Three PARPis are approved for ovarian and/or breast cancers. All PARPis comprise a nicotinamide moiety (red), which binds the $\beta-N A D^{+}$acceptor site in PARP-1 and PARP-2. PARPis differ by their PARPtrapping potency. (B) PARPis are potential substrates for the drug efflux pumps (PgP/ABCB1), which limit cellular accumulation. Binding of the PARPis to the NAD+ site of PARP-1 and PARP-2 (red circle) results both in catalytic inhibition by competitive inhibition of $\beta$-NAD binding and in PARP trapping by a proposed reverse allosteric mechanism (5). Trapping can be mitigated by increased PARP-1 and PARP-2 auto-PARylation due to high NAD+ synthesis by nicotinamide phosphoribosyltransferase (NAMPT) or enhanced by PAR removal by poly(ADP-ribose) glycohydrolase (PARG). Trapping of PARP-1 and PARP-2 damages replicating cells. Cells can alleviate this effect by stabilizing replication forks through BRCA2 and other factors of the Fanconi anemia pathway (FANC) or suppression of the polycomb complex (EZH2). Damaged cells can also repair the broken forks by homologous recombination (HR) involving BRCA1 or BRCA2 or commit themselves to death through Schlafen 11 (SLFN11). Inactivation of 53BP1 and REV7 reactivates HR. Red symbols define determinants of response to the PARPis. Blue symbols signify resistance to PARPis. Synthetic lethality (4) occurs in cancer cells deficient for the resistance factors highlighted in blue. BRCA1 and BRCA2 were the first resistance factors identified and led to the approval of PARPis as monotherapy.

clinical responses that sensitivity and resistance to PARPis is determined by factors beyond tumor DNA repair status (Figure 1).

\section{Determinants of response and resistance to monotherapy PARPis}

The first identified determinants of response to PARPi were the presence of deleterious BRCA1 and BRCA2 mutations $(14,15)$, which substantially sensitize cancer cells to PARPis $(5,14,15)$ and render such cells defective in HR, such as occurs in HRD cancers. It is increasingly clear that $\mathrm{HR}$ repair is a multifactorial process that involves many proteins beyond BRCA1 and BRCA2. Indeed, at the beginning of $\mathrm{HR}$, the ends of the DNA double-strand breaks need to be processed by nucleases (MRE11, CtIP, DNA2, exonuclease 1) to generate $3^{\prime}$ single-stranded DNA (ssDNA) tails. These ssDNA tails need to be coated by RAD51 to form presynaptic filaments, which then invade a duplex region of intact DNA that is homologous to the broken DNA, forming D-loops that require chromatin remodeling and adjustment of DNA supercoiling. The invading DNA needs to be copied by DNA polymerase(s) and ligated before restoration of the original DNA sequence. Finally, after dissociation, the repaired DNA can move back to its normal nuclear position (territory). The precise regulation and factors involved in many of these steps are still unknown, explaining why it remains difficult to comprehensively identify HRD based on current genomic analyses. Furthermore, multiple different mutations can lead to loss of BRCA1 or BRCA2 function, and scoring such mutations can be ambiguous. An alternative to single gene mutation, deletion, or methylation analyses is to use HRD genomic signatures. However, HRD genomic signatures may only represent so-called mutational genomic scars, which persist while the cancer cells reactivate $\mathrm{HR}$. For instance, inactivation of 53BP1 (16), REV7 (17), and EZH2 (18) in BRCA1- or BRCA2-deficient cells can reactivate $\mathrm{HR}$ and confer resistance to PARPis (Figure 1).

Other important determinants of the response downstream of PARP trapping are the ability of cells to stabilize replication forks (5) or, alternatively, to irrevers- 
ibly arrest replication in response to replication stress (19). In addition to their role in HR repair, BRCA2 and other Fanconi anemia-associated genes act to stabilize replication forks, whereas EZH2 tends to promote replication fork breakage (collapse) by engaging MUS81 endonuclease at such forks (Figure 1) (18). In addition, recent studies demonstrate that stressed replication forks can induce irreversible cell-cycle arrest by engaging Schlafen 11 (SLFN11) with the replication helicase complexes, and blocking replication by opening chromatin through the ATPase activity of SLFN11 (20).

\section{Implications of a PARP-1 tracer}

Protein levels of PARP-1 and PARP-2 are also determinants for PARPi sensitivity, because PARP-1 and PARP-2 are required as substrates for PARP trapping. In this issue, a study by Makvandi and coworkers (21) confirms the requirement of PARP-1 for the cytotoxicity of PARPis (Figure 1) and shows that low PARP-1 expression is among the potential causes of resistance to PARPis. The Makvandi et al. study (21) is primarily aimed at providing a way to determine the level of PARP-1 in tumors by a novel imaging procedure. The authors used $\left[{ }^{18} \mathrm{~F}\right]$ FluorThanatrace $\left(\left[{ }^{18} \mathrm{~F}\right] \mathrm{FTT}\right)$ for PET. $\left[{ }^{18} \mathrm{~F}\right] \mathrm{FTT}$ has previously been demonstrated to result in highly specific tracer uptake in animal models and humans $(22,23)$. MicroPET studies in a preclinical patient-derived xenograft (PDX) mouse model showed high tracer uptake in tumors relative to normal tissue. Blockade of radiotracer uptake following pretreatment with olaparib was used to confirm specificity of $\left[{ }^{18} \mathrm{~F}\right] \mathrm{FTT}$ for PARP-1. In a clinical trial of patients with epithelial ovarian cancer who underwent surgical debulking or biopsy, $\left[{ }^{18} \mathrm{~F}\right] \mathrm{FTT}$ localized to areas of known cancer. Moreover, $\left[{ }^{18} \mathrm{~F}\right] \mathrm{FTT}$ demonstrated a wide dynamic range of uptake, correlating with in vitro measures of PARP-1 expression.

The study by Makvandi et al. provides proof-of-principle that a noninvasive imaging procedure can assess PARP-1 levels and/or activity. Whether this approach will be useful in determining which patients may benefit from PARPi therapy warrants further study. PARP-1 is an abundant protein in the nucleus and a fraction of it is trapped by PARPis. Hence, it is not clear how much reduced expression of PARP-1 could contribute to the reduction of PARP trapping and resistance to PARPis. In the cancer cell line databases CellMiner CDB (http:// discover.nci.nih.gov/cellminercdb), NCI60 (https://dtp.cancer.gov/discovery_dev elopment/nci-60/), Cancer Cell Line Encyclopedia (https://portals.broadinstitute. org/ccle), and Genomics of Drug Sensitivity in Cancer (http://www.cancerrxgene. org/), none of the approximately 1,000 cell lines is null for PARP-1, suggesting that PARPi resistance due to PARP-1 deficiency is not expected in tumors. Data showing a positive correlation between PARP-1 expression level and sensitivity to PARPis are necessary to utilize the methods of Makvandi et al. in the clinic. Archived tumors could be retrospectively examined for PARP-1 expression and response to PARPis. It would also be important to test $\left[{ }^{18} \mathrm{~F}\right] \mathrm{FTT}$ PET in parallel with PARPis in clinical trials that stratify patients based on other known determinants of PARPi response. The effect of heterogeneity of $\left[{ }^{18} \mathrm{~F}\right]$ FTT-based PARP-1 expression in terms of tumor responses also warrants further investigation. $\left.{ }^{[18} \mathrm{F}\right]$ FTT-based determination of PARP-1 expression could also allow monitoring for the emergence of resistance mediated by downregulation of PARP-1.

In conclusion, multiple effectors and molecular signatures should be considered for the prediction of responders and nonresponders to PARPis, including DNA repair and replication defects, expression of SLFN11, and hyperactive drug efflux pumps. The novel approach described by Makvandi and coworkers will enable us to monitor not only PARP-1 expression levels in patient tumors but also the penetration of PARPis into tumors, which can provide us with useful information for the selection of patients who may benefit from PARPi therapy.

\section{Acknowledgments}

Our studies are supported by the Center for Cancer Research, the intramural program of the National Cancer Institute, NIH (BC 006150 and 011793).

Address correspondence to: Yves Pommier, Building 37, Room 5068, NIH, Bethesda, Maryland 20892, USA. Phone: 240.760.6142; Email: pommier@nih.gov.
1. Leidecker $\mathrm{O}$, et al. Serine is a new target residue for endogenous ADP-ribosylation on histones. Nat Chem Biol. 2016;12(12):998-1000.

2. Daniels CM, Ong SE, Leung AK. The promise of proteomics for the study of ADP-ribosylation. Mol Cell. 2015;58(6):911-924.

3. Pommier Y, O'Connor MJ, de Bono J. Laying a trap to kill cancer cells: PARP inhibitors and their mechanisms of action. Sci Transl Med. 2016;8(362):362ps17.

4. Lord CJ, Ashworth A. PARP inhibitors: synthetic lethality in the clinic. Science. 2017;355(6330):1152-1158.

5. Murai J, et al. Trapping of PARP1 and PARP2 by clinical PARP inhibitors. Cancer Res. 2012;72(21):5588-5599.

6. Robson M, et al. Olaparib for metastatic breast cancer in patients with a germline BRCA mutation. NEngl J Med. 2017;377(6):523-533.

7. Coleman RL, et al. Rucaparib maintenance treatment for recurrent ovarian carcinoma after response to platinum therapy (ARIEL3): a randomised, double-blind, placebo-controlled, phase 3 trial. Lancet. 2017;390(10106):1949-1961.

8. Mirza MR, et al. Niraparib maintenance therapy in platinum-sensitive, recurrent ovarian cancer. N Engl J Med. 2016;375(22):2154-2164.

9. Ledermann J, et al. Olaparib maintenance therapy in platinum-sensitive relapsed ovarian cancer. N Engl J Med. 2012;366(15):1382-1392.

10. Murai J, et al. Stereospecific PARP trapping by BMN 673 and comparison with olaparib and rucaparib. Mol Cancer Ther. 2014;13(2):433-443.

11. Murai J, et al. Rationale for poly(ADP-ribose) polymerase (PARP) inhibitors in combination therapy with camptothecins or temozolomide based on PARP trapping versus catalytic inhibition. J Pharmacol Exp Ther. 2014;349(3):408-416.

12. Liu SK, Coackley C, Krause M, Jalali F, Chan N, Bristow RG. A novel poly(ADP-ribose) polymerase inhibitor, ABT-888, radiosensitizes malignant human cell lines under hypoxia. Radiother Oncol. 2008;88(2):258-268.

13. Mirza MR, et al. Niraparib maintenance therapy in platinum-sensitive, recurrent ovarian cancer. N Engl J Med. 2016;375(22):2154-2164.

14. Farmer H, et al. Targeting the DNA repair defect in BRCA mutant cells as a therapeutic strategy. Nature. 2005;434(7035):917-921.

15. Bryant HE, et al. Specific killing of BRCA2deficient tumours with inhibitors of poly(ADP-ribose) polymerase. Nature. 2005;434(7035):913-917.

16. Jaspers JE, et al. Loss of 53BP1 causes PARP inhibitor resistance in Brca1-mutated mouse mammary tumors. Cancer Discov. 2013;3(1):68-81.

17. Xu G, et al. REV7 counteracts DNA doublestrand break resection and affects PARP inhibition. Nature. 2015;521(7553):541-544.

18. Rondinelli B, et al. EZH2 promotes degradation of stalled replication forks by recruiting MUS81 through histone $\mathrm{H} 3$ trimethylation. Nat Cell Biol. 2017;19(11):1371-1378.

19. Murai J, et al. Resistance to PARP inhibitors by SLFN11 inactivation can be overcome by ATR inhibition. Oncotarget. 2016;7(47):76534-76550. 
20. Murai J, et al. SLFN11 blocks stressed replication forks independently of ATR. Mol Cell. 2018;69(3):371-384.e6.

21. Makvandi M, et al. A PET imaging agent for evaluating PARP-1 expression in ovarian cancer.
J Clin Invest. 2018;128(5):2116-2126.

22. Zhou D, et al. Synthesis, $\left[{ }^{18} \mathrm{~F}\right]$ radiolabeling, and evaluation of poly (ADP-ribose) polymerase-1

(PARP-1) inhibitors for in vivo imaging of PARP-1 using positron emission tomography. Bioorg Med
Chem. 2014;22(5):1700-1707.

23. Edmonds CE, et al. [(18)F]FluorThanatrace uptake as a marker of PARP1 expression and activity in breast cancer. Am J Nucl Med Mol Imaging. 2016;6(1):94-101. 